\title{
LINKING THE CAROLINA CURRICULUM FOR PRESCHOOLERS WITH SPECIAL NEEDS TO THE ICF-CY
}

\author{
Susana Castro, Ana Isabel Pinto and Mónica Maia
}

\section{Introduction}

The aim of this article is to document and discuss the utility of linking a curriculum based developmental measure, designed for the assessment of preschoolers with mild to severe special needs, to the codes of an international classification system developed by the World Health Organization (WHO). This classification system, the International Classification of Functionality, Disability and Health (ICF; WHO, 2001), was developed to serve as a common language among professionals of diverse disciplines, based on the biopsychosocial model of disability (Frankel et al., 2003). The results of this study are discussed in relation to the characteristics and use of classifications, which stem from different models of disability. The discussion contributes to an increased understanding of (1) the association between the content of developmental measures and an international classification of child functioning, and (2) its utility in documenting and planning assessmentintervention procedures for children attending inclusive preschool settings.

The concept of disability varies,

\section{*Susana Castro, Master in Psychology; Doctoral fellow at the Faculty of Psychology and Educational Sciences \\ Porto University, Center of Developmental Psychology and Child Education, Rua Dr. \\ Manuel Pereira da Silva 4200-392 Porto, Portugal \\ Tel: +351-916254028 Email: pdpsi08030@fpce.up.pt}

Ana Isabel Pinto, PhD in Educational Psychology; Professor of Psychology and Early Childhood Intervention

Faculty of Psychology and Educational Sciences of Porto University, Portugal and member of the Transatlantic Consortium for Global Education and Developmental Studies (GEDS),

Rua Dr. Manuel Pereira da Silva 4200-392 Porto, Portugal

Tel: +351-226079700 Email: ana@fpce.up.pt

Mónica Maia, Graduation in Occupational Therapy; Doctoral fellow at the Faculty of Psychology and Educational Sciences

Porto University, Center of Developmental Psychology and Child Education, Rua Dr.

Manuel Pereira da Silva 4200-392 Porto, Portugal

Tel: +351-916254028 Email: pdpsi08030@fpce.up.pt

${ }^{\star}$ For correspondence 
depending on the theoretical framework used to define it. Since the beginning of the twentieth century, the concept of disability has been formulated according to a medical-model approach, which focuses on categorizing the person based on symptoms and defining diagnostic groups of individuals with the purpose of establishing eligibility. According to this model, the cause of disability lies within the individual and the corresponding interventions frequently involve medical treatment (Simeonsson, 2006). The International Classification of Diseases (ICD-10; WHO, 1994) was developed based on this model, providing codes that describe diagnostic categories. By the 1960 's, with the emergence of the social model of disability, also named "Minority Groups Model", the emphasis shifted from the individual to the society as the source of disability. We may thus posit that society is responsible for providing the resources required for optimized individual functioning, regardless of impairment. This shift in approaching disability has had a tremendous impact on intervention design: the intervention target has been transferred from the person to aspects of its' environment, in order to enhance the context according to the needs of the individual. By the 1990's, another, complimentary, disability perspective emerged - the functional model of disability, emphasizing the dynamic process of interaction between the individual and the environment. This is to say, the way in which the environment fits the individual and also the way in which the individual accommodates to the environmental resources should be considered in the assessment-intervention process (Simeonsson, 2006). This approach to disability is based on ecological and transactional approaches to development, and assumes a systems perspective, considering both the individual and its respective environment as inseparable elements in the developmental process. In fact, the Transactional Model (Sameroff, 2009), conceptualizes development as a dynamic process of continuous and reciprocal interactions between person and context over time, effecting changes in children's development, as well as in their various environmental settings. The concept of transaction proposed by Sameroff and Chandler (1975) underlines the dynamic nature of internal and external processes and has become central to understanding the interplay of nature and nurture in the development of both positive and negative outcomes for children. Within this process, protective factors and risk factors at both the individual and/or contextual levels influence the course of development. This is done either by allowing the development of coping strategies to deal with stress, adversity, and trauma in an adaptive and functional way, or by restricting the ability of the person to deal with adverse life circumstances.

According to the bio-ecological approach (Bronfenbrenner, 2001; Bronfenbrenner and Morris, 1998), the study of human development is marked by a focus on understanding the dynamic change resulting from the interaction and mutual influence between the developing person and the integrated, multilevel ecology of the contexts in which the individual lives in. This perspective of development is particularly relevant in the Early Childhood Intervention (ECI) field, as it considers the assorted contexts in which the individual is embedded, underlining the influence of proximal and distal processes of interaction between the child and the context. Within developmental processes, the authors also refer to the particular influence of 
bio-ecological requests, strengths and resources of the individual. Therefore, the transactional and bio-ecological perspectives provide developmental frameworks that emphasize the proactive and contextualized nature of human functioning.

The biopsychosocial model, which stems from the two aforementioned models, proposes a theoretical framework directed at accounting for the different dimensions of human functioning, and is illustrated by the International Classification of Functionality, Disability and Health (ICF; WHO, 2001) taxonomy. The ICF is the most current publication by the International Classification of the $\mathrm{WHO}$, aimed at overcoming the traditional medical model of categorizing disability according to diagnostic groups. The ICF proposes taxonomy of functionality dimensions, classifying characteristics of functioning rather than people themselves. Also, this taxonomy provides a standard language to approach functioning across different areas of research, and practice, as well as across cultures. The ICF taxonomy comprises three different areas of human functioning, in line with the biopsychosocial model: (i) body functions and body structures; (ii) activities and participation; (iii) environmental factors; and each area comprises a detailed dimension of functioning. A version of the ICF for Children and Youth (ICF-CY) was recently published (ICF-CY; WHO, 2007). The Youth version of the ICF is important, as children's engagement in daily activities varies from that of adults in form, function and context. In addition, children's body functions and structures continue to undergo developmental changes, an aspect recognized in the current edition, in which several codes were added to describe specific aspects of children's functioning (Lollar and Simeonsson,
2005). The ICF-CY enables the standard description of individual characteristics of functioning, regarding the individual, independent of the diagnostic category in which that person might have been included. In fact, the ICF and the ICD-10 provide a complementary way to describe functioning, according to the conceptual model of the international classification system. As an example, we may consider several different children diagnosed with F84 (Pervasive Developmental Disorders), according to the ICD-10 diagnostic criteria but each of them may present varied aspects of functioning, which may then be described using the ICF-CY common language. This integrative use of classification would provide data useful for professionals from different disciplines for planning integrated and individualized assessment and intervention (Lollar and Simeonsson, 2005).

Despite all the mentioned advantages of the ICF-CY as a classification system in documenting individualized functionality profiles and in planning assessment and intervention, the ICF$\mathrm{CY}$ is not an assessment instrument. The need for assessment measures that are compatible with the ICF-CY description of functioning is widely recognized, and has created a unique line of investigation employing the ICF model (Xiong and Hartley, 2008). To conduct the linkage process between ICF components and the assessment measurement's items, a set of rules were published which have been extensively applied in the literature (Cieza et al., 2005). Despite the recognition of such need, the majority of studies already published within this particular line of research concern mainly health measures or health-related measures. Studies linking developmental measures with the ICF-CY classification system are still rare, and the need for data based on authentic 
assessment procedures enabling the documentation of children's functioning for educational and transition planning, have also been widely recognized (Bagnato et al., 2010).

In Portugal, the ICF was recently inducted into the law as a tool to support eligibility identification for special education and early childhood intervention services. For a successful implementation of the ICF in educational services, more research is needed in order to fill the lack of resources that could guide this process, namely, instruments linked with the classification, advanced training for educational professionals, and guidelines for the application of the ICF in specific settings. To this point, Castro and Pinto (in prep.) report finding a substantial number of educational profiles were developed solely based, either on teachers' informal assessments, or on clinical information provided through reports from other professionals other than the preschool and support teachers. Moreover, it was found that one of the most widely used measures to assess functioning of children with disabilities in Portuguese preschool settings is the Carolina Curriculum for Preschoolers with Special Needs (CCPSN)(Johnson-Martin et al., 1990).

The aim of the present study is to identify the ICF-CY dimensions of functionality that might be assessed with the CCPSN by linking this instrument's items with the ICF-CY classification system, as well as to discuss the implications of using the ICF-CY to describe assessment outcomes obtained with CCPSN.
Bagnato and collaborators (2010) argue the need for a common language in ECI that may translate authentic assessment using different developmental dimensions involved in young children's functioning, into a profile that mirrors their real participation in daily settings, thus achieving a systemic thinking within a transdisciplinary approach. In Portuguese ECI services, this argument is especially relevant, considering teacher mobility and the difficulty in planning and documenting educational transitions, particularly for children with developmental disabilities.

The present study has an exploratory nature and intends to add knowledge to this new area of research that may be of use in ECI and special education fields. It is also expected that study results may inspire further efforts in testing the utility of the mapping between measures and the ICF-CY, as well as in developing tools to facilitate transdisciplinary narratives.

\section{Method}

Two independent researchers (the principal investigator and a research assistant) linked the CCPSN items with the ICF-CY classification system using content analysis strategies.

As previously described, the ICF taxonomy comprises three chapters, in line with the biopsychosocial model of functioning: (i) body functions and body structures; (ii) activities and participation; (iii) environmental factors ${ }^{1}$. Each chapter provides detailed dimensions of function-

\footnotetext{
${ }^{1}$ According to the ICF model definitions, the Body Functions are the physiological functions of the organic system, including psychological functions; the Body Structures are the anatomic part of the body, such as organs, members and its components; An Activity is the execution of a task or action, by an individual; Participation is the involvement of the individual in a real life situation; the Environmental Factors comprise the physical, social and attitudinal environment in which people live (WHO, 2001).
} 
ing and each dimension is identified with an alphanumeric code ${ }^{2}$. Following this code, the ICF universal qualifier, describing the magnitude of the problem in a specific functionality dimension, using a 5 point scale which starts from 0 (absence of impairment, difficulty or restriction) to 4 (total impairment, difficulty or restriction) is added. In relation to the environmental factors, the universal qualifier describes the level in which a specific factor is a barrier or a facilitator to functioning.

The CCPSN is a curriculum based observational measure designed for the assessment of children with mild to severe disabilities from 2 to 5 years of age. The curriculum itself is divided into 22 logical teaching sequences covering five developmental domains: cognition, communication, social adaptation, fine motor, and gross motor, in a total of 418 items.

Deductive content analysis (Elo and Kyngas, 2007) was used to make inferences from textual data by grouping together similar types of utterances and ideas, since the final categories - the ICF-CY functionality domains - were known to the researchers prior to the content analysis procedure. The process of analysis has followed a set of sequential steps using the published linking rules (Cieza et al., 2005) on the identification of meaningful concepts. Both the principal investigator and a research assistant had previous knowledge, as well as extended experience in using the ICFCY classification system, as stated in the first linking-rule recommendation. These linking rules have been extensively used in linking health status measures or health related measures to the ICF (Starrost et al., 2008). An addendum to these rules was developed for the purpose of the present study, due to the evidence that, the CCPSN, being a developmental measure, has items with a much broader content than the health status measures, which turned the definition of what should be a meaningful concept into a challenging demand. Therefore, the two researchers conducting the linking process defined the following rules in addition to the published linking rules (Cieza et al., 2005):

(a) Only meaningful concepts related to the purpose of assessment were coded;

In the definition of what should be a meaningful concept, the two researchers conducting the linking process first considered the purpose of assessment for each item. Whenever the researchers identified meaningful concepts not related to the item's purpose of assessment, that concept was not coded, otherwise there would be a large amount of information that did not directly answer the purpose of assessment. To exemplify this situation, if the researchers had to identify the meaningful concepts in the item "The child points to the hand where the object was hidden", the information relating to the child's ability to point in order to communicate was not considered, as the assessment purpose of the item is related to the attention performance of the child. Reading of the whole item, prior to splitting it into meaningful concepts should provide the most precise code, according to its purpose for assessment "directing attention" ( $\left.\mathrm{d} 161^{3}\right)$.

(b) If there were two ICF-CY codes that

${ }^{2}$ The first character of this alphanumeric code is a letter by which we identify the ICF chapter: $b$ for Body Functions; s for Body Structures; d for Activities and Participation, meaning domain; e for Environmental factors. The following characters are numbers relating to specific functionality domains within the chapter.

${ }^{3}$ Alphanumeric codes of ICF-CY taxonomy, relating to the chapter of Activities ad Participation. 
the researchers found relevant to define the purpose of assessment of the item, the two codes should be added;

In the process of decision-making regarding the global meaning of the item using the most precise ICF-CY category to define it, the researchers decided it was possible to add more than one ICF-CY code, as long as the purpose of assessment of that item included more than one functionality dimension. As an example, in the item "The child recalls a casual event", both "rehearsing" (d135) and "directing attention" (d161) were added, as both functionality dimensions are essential to describe the purpose of assessment of the item "attention and memory performance".

(c) If the item described aspects of children's performance that reveal the state of related body functions, the respective body function was also considered

In young children, the assessment of mental functions is frequently conducted through the observation of conceptually related behaviours, performance, and/ or forms of participation. The assessment of "pure" function in young children, decontextualized and conducted in a neutral and artificial environment, is often unviable, due to the maturation status of the child that cannot respond to complex laboratory instructions. As Bagnato (2010) stated, functional assessment is best conducted in context; as the child's performance in context will give us hints on the status of the underlying functions associated with a specific form of participation. While conducting the linking process, whenever the item represented an activity or a form of participation that was conceptually related to a specific body function, the body function was also coded.

After defining the additional rules that guided the linking process, a training phase of the linking process was conducted with the two researchers conducting the linking process, in order to ensure that both were using similar criteria when applying the rules. This training was conducted with $5 \%$ of the total items of the CCPSN (total of 21 items), randomly selected in equal number from each of its developmental sections, to ensure that the rules were correctly applied in each section of the instrument. In this training phase, discrepant responses were discussed according to the defined rules, to clarify criteria and obtain consensus in coding for those items.

In the next phase of the linking process the two researchers independently linked the CCPSN items with the ICFCY classification system. Inter-rater agreement was then calculated using Kappa Coefficient (Cohen, 1960) ${ }^{4}$. For items with discrepant coding, final coding resulted from the discussion between the two researchers about the criteria used in the items where disagreement was obtained.

\section{Results}

The degree of agreement between the two researchers when linking CCPSN items with the ICF-CY had a mean Kappa

${ }^{4}$ Kappa coefficient, as introduced by Cohen in 1960, is a reliability index to measure the proportion of interrater agreement in nominal scales, eliminating the effect of agreement by chance (McCartney \& Rosenthal, 2000). Kappa values vary between -1 (total disagreement) and 1 (perfect agreement); a vale of zero means that the fond agreement is exactly due to chance. Kappa values have been classified according to the agreement robustness (Landis \& Koch, 1977); .01 $<\mathrm{k}<.20$ - slight; .21 $<\mathrm{k}<.40$ reasonable; $.41<\mathrm{k}<.60$ - moderate; $.61<\mathrm{k}<.80$ - substantial; $.81<\mathrm{k}<1.00$ - almost perfect. 
value of .43 for the 418 items (TABLE I), indicating a moderate level of agreement (Landis and Koch, 1977). It is notable that the proportion of agreement between the two raters is relatively higher for the body functions' domains (Kappa: 0.61) than for the activities and participation's domains (Kappa: 0.46). Only 28.1\% of the items were linked with total disagreement between the two raters, and $71.3 \%$ of the CCPSN items were linked to the ICF-CY with some level of agreement. Among the latter, we identified the following levels of agreement: (a) items in which the two researchers had complete agreement concerning the exact code selected; (b) items in which the researchers agreed in the main functionality domain to be linked, but disagreed at the level of ICFCY specification, within that domain; (c) items in which each researcher used more than one code, but only agreed on one of them; (d) items where the two previous conditions, ' $b$ ' and ' $c$ ', were observed at the same time (TABLE II).

In relation to the ICF-CY functionality domains that are covered by the CCPSN items, the body functions $(87.1 \%)$ and activities and participation (97.6\%) domains were nearly commensurate (TABLE III). This phenomenon may illustrate a relationship between certain components of these two chapters of the ICF-CY (TABLE IV). For some of the body functions domains, the relationship with the activities and participation dimensions can be conceptually established prior to the linking process. For example, the body function domain of language functions (b167) has a relationship that can be

TABLE I

Average of inter-rater agreement with Kappa Cohen Coefficient*

\begin{tabular}{|c|c|c|}
\hline Mean Total Kappa* & Mean Kappa in Body Functions* & $\begin{array}{c}\text { Mean Kappa in Activities \& } \\
\text { Participation }\end{array}$ \\
\hline .46 (moderate) & .61 (good) & .43 (moderate) \\
\hline
\end{tabular}

*Kappa values vary between -1 (total disagreement) and 1 (perfect agreement); a value of zero means that the fond agreement is exactly due to chance. Kappa values have been classified according to the agreement robustness (Landis and Koch, 1977); .01 <k $<.20$ - slight; $.21<k<.40$ - reasonable; $.41<k<.60$ - moderate; $.61<k<.80$ - substantial; . $81<k<1.00$ - almost perfect

TABLE II

Percentage of different levels of agreement

\begin{tabular}{|c|c|c|c|c|}
\hline \multicolumn{5}{|c|}{$\mathrm{N}=418$ items } \\
\hline $\begin{array}{l}\text { (a) Complete } \\
\text { Agreement in } \\
\text { the exact code } \\
\text { assigned to item }\end{array}$ & $\begin{array}{l}\text { (b) Agreement in } \\
\text { code assigned } \\
\text { to item/ } \\
\text { Disagreement at } \\
\text { the level of ICF- } \\
\text { CY soecification }\end{array}$ & $\begin{array}{l}\text { (c) Agreement } \\
\text { in at least one } \\
\text { code assigned } \\
\text { to item and } \\
\text { disagreement } \\
\text { in another code } \\
\text { assigned to } \\
\text { same item }\end{array}$ & $\begin{array}{l}\text { (d) Agreement } \\
\text { conditions (b) } \\
\text { and (c) are met }\end{array}$ & $\begin{array}{l}\text { Total } \\
\text { Disagreement in } \\
\text { codes assigned } \\
\text { to one item }\end{array}$ \\
\hline $220(52.6 \%)$ & 35 & \multirow{2}{*}{43} & \multirow{3}{*}{6} & \multirow{4}{*}{$114(28.1 \%)$} \\
\hline 255 & $61 \%)$ & & & \\
\hline \multicolumn{3}{|c|}{$298(71.3 \%)$} & & \\
\hline \multicolumn{4}{|c|}{$304(72.7 \%)$} & \\
\hline
\end{tabular}


TABLE III

Percentage of ICF-CY domains found in CCPSN

\begin{tabular}{l|l} 
\% Activities \& Participation & \% Body Functions
\end{tabular}

\begin{tabular}{l|l}
$408(97.6 \%)$ & $364(87.1 \%)$
\end{tabular}

TABLE IV

Frequency of Body Functions linked with the CCPSN and related Activities and Participation domains

\begin{tabular}{|c|c|}
\hline Body Functions & Activities and Participation chapter \\
\hline b140 Attention Functions x 24 & \multirow[t]{3}{*}{ d1 (Learning and applying knowledge) } \\
\hline b144 Memory Functions x 24 & \\
\hline b117 Intellectual functions x 47 & \\
\hline b156 Perceptual functions $\times 30$ & $\begin{array}{l}\text { d1 (Learning and applying knowledge) } \\
\text { d4 (mobility) }\end{array}$ \\
\hline b164 Higher level cognitive functions x 12 & $\begin{array}{l}\text { d1 (Learning and applying knowledge) } \\
\text { d3 (Communication) } \\
\text { d8 (Major Life areas) }\end{array}$ \\
\hline b167 Mental functions of language $\times 86$ & $\begin{array}{l}\text { d1 (Learning and applying knowledge) } \\
\text { d3 (communication) }\end{array}$ \\
\hline b126 Temperament and personality functions $x 10$ & $\begin{array}{l}\text { d2 (General tasks and demands) } \\
\text { d5 (Self-care) }\end{array}$ \\
\hline b122 Psychosocial functions $\times 21$ & $\begin{array}{l}\text { d1 (Learning and applying knowledge) } \\
\text { d3 (Communication) } \\
\text { d8 (Major Life areas) } \\
\text { d2 (General tasks and demands) } \\
\text { d5 (Self-care) } \\
\text { d7 (Interpersonal relationships) }\end{array}$ \\
\hline
\end{tabular}

conceptually related to the activities and participation speaking domain (d330). This relationship, however, is not as clear for other body functions domains, as such functions may underlie different activity and participation domains (e.g., b126 temperament and personality functions). The relationship between temperament and performance is more complex and dependent on personal factors than the aforementioned relationship between language functions and speaking, or between cognitive functions (b164) and thinking (d163).

Among the body functions domains that are covered by the CCPSN, the intellectual functions (b122), the psychomotor functions (b147) and the expression of spoken language functions (b16710) were the most frequently coupled with the CCPSN items (TABLE IV). In items concerning the activities and participation domains, the codes most frequently assigned were related to fine hand use (d440), jumping (d4553) and acquiring basic concepts (d1370) (TABLE $\mathrm{V})$. CCPSN does not cover environmental factors or body structures, as the ICF does. 
TABLE V

Frequency of Activities and Participation domains linked with the CCPSN and the frequency of linkage per code

\begin{tabular}{|c|c|}
\hline ICF-CY domain (Activities and Participation) & $\begin{array}{l}\text { Frequency } \\
\text { of items }\end{array}$ \\
\hline d130 Imitation & 18 \\
\hline d1311 Learning through simple action with a single object & 9 \\
\hline $\begin{array}{l}\text { d1312 Learning through actions by relating two or more objects with regard to specific } \\
\text { features }\end{array}$ & 3 \\
\hline d1313 Learning through symbolic play & 3 \\
\hline d1314 Learning through pretend play & 1 \\
\hline d132 Acquiring information & 3 \\
\hline d1330 Acquiring simple words or meaningful symbols & 11 \\
\hline d1331 Combining words into phrases & 4 \\
\hline d1332 Acquiring syntax & 20 \\
\hline d135 Rehearsing & 11 \\
\hline d1370 Acquiring basic concepts & 28 \\
\hline d1371 Acquiring complex concepts & 8 \\
\hline d1450 Acquiring skills to use writing implements & 3 \\
\hline d1502 Acquiring skills in using basic operations & 2 \\
\hline d161 Directing attention & 9 \\
\hline d163 Thinking & 19 \\
\hline d1630 Pretending & 4 \\
\hline d1631 Speculating & 11 \\
\hline d2400 Handling responsibilities & 3 \\
\hline d250 Managing Behavior & 7 \\
\hline d2501 Responding to demands & 2 \\
\hline d2503 Acting predictability & 3 \\
\hline d3102 Comprehending complex spoken messages & 16 \\
\hline d3150 Communicating with - receiving - body gestures & 1 \\
\hline d330 Speaking & 19 \\
\hline d332 Singing & 1 \\
\hline d3350 Producing Body Language & 2 \\
\hline d3352 Producing drawings and photographs & 7 \\
\hline d350 Conversation & 4 \\
\hline d3500 Starting a conversation & 1 \\
\hline d3600 Using telecommunication devices & 1 \\
\hline d4106 Shifting the body center of gravity & 7 \\
\hline d4154 Maintaining a standing position & 1 \\
\hline d4351 Kicking & 6 \\
\hline
\end{tabular}




\begin{tabular}{|c|c|}
\hline d440 Fine Hand Use & 44 \\
\hline d4400 Picking up & 3 \\
\hline d4401 Grasping & 2 \\
\hline d4402 Manipulating & 31 \\
\hline d4453 Turning or Twisting the hands or arms & 7 \\
\hline d4454 Throwing & 5 \\
\hline d4455 Catching & 5 \\
\hline d450 Walking & 14 \\
\hline d4500 Walking short distances & 3 \\
\hline d4502 Walking on different surfaces & 7 \\
\hline d4551 Climbing & 15 \\
\hline d4552 Running & 4 \\
\hline d4553 Jumping & 31 \\
\hline d465 Moving around using equipment & 1 \\
\hline d5100 Washing body parts & 2 \\
\hline d5102 Drying oneself & 2 \\
\hline d5201 Caring for teeth & 1 \\
\hline d5202 Caring for hair & 1 \\
\hline d5205 Caring for nose & 1 \\
\hline d53000 Indicating need for urination & 1 \\
\hline d53001 Caring out urination appropriately & 4 \\
\hline d53010 & 2 \\
\hline d53011 Indicating need for defecation & 56 \\
\hline d5501 Caring out eating appropriately & 7 \\
\hline d560 Drinking & 4 \\
\hline d540 Dressing & 10 \\
\hline d5400 Putting on clothes & 4 \\
\hline d5401 Taking off clothes & 3 \\
\hline d5403 Taking off footwear & 2 \\
\hline d571 Looking after one's safety & 2 \\
\hline d6300 Preparing simples meals & 1 \\
\hline d710 Basic interpersonal interactions & 7 \\
\hline d6406 Helping to do Housework & 1 \\
\hline d7100 Respect and warmth in relationships & 2 \\
\hline d71040 Initiating social interactions & 1 \\
\hline d7106 Differentiation of familiar persons & 3 \\
\hline d7203 Interacting according to social rules & 3 \\
\hline d7504 Informal relationships with peers & 3 \\
\hline d880 Engagement in play & 6 \\
\hline d8802 Parallel play & 1 \\
\hline d8803 Shared cooperative play & 4 \\
\hline Total & 503 \\
\hline
\end{tabular}




\section{Discussion and Practical Application}

The present study aimed to contribute to the improvement of the assessmentintervention process of young children with special needs. This was done within the context of the Portuguese special education law by providing increased understanding of the association between the content of a widely used developmental measure, the CCPSN, and an international classification of child functioning - the ICF-CY. As the current Portuguese Special Education and Early Childhood Intervention laws recommend the use of the ICF-CY, such increased knowledge may be useful, not only in documenting and planning assessmentintervention procedures, but also in reporting children's functionality profiles and, consequently, in defining criteria for the eligibility of these children to the support services.

The lack of measures to document children's functionality, as well as the lack of training for special education professionals on the use of the ICF-CY have been widely documented. In this study, the CCPSN was identified and selected from measures most currently used in early childhood intervention in Portugal, to be linked with the ICF-CY. This linkage provides several advantages that address the aim of the study, as it: (a) enables the linkage between assessment and intervention - as a curriculum based measure CCPSN provides data for assessment procedures and for planning interventions; the need for linkage between assessment and intervention is widely recommended, in order to give a sense of constancy and predictability to the child's improvement of performance (Bagnato, 2010), (b) the linkage of CCPSN with the ICF-CY provides information on the functionality domains that are covered by this instrument, according to the ICFCY taxonomy, which may be useful for daily special education professionals work, as the assessment conducted with the CCPSN may be documented trough the ICF-CY taxonomy; (c) assessments conducted with the CCPSN in different preschool settings, individual children, or in a group of children attending an early childhood intervention programme may also be compared using a common language, once the ICF-CY was empirically linked with this measure; (d) linkage of the CCPSN with the ICF-CY may also be useful for statistical reports on the functionality profiles of young children with special needs included in a regular preschool classroom, using a standard language.

The process of linking CCPSN with the ICF-CY also presented several challenges. The strategies used to conduct this process were deductive content analysis and the appropriate linking rules (Cieza et al., 2005). Since these are qualitative strategies, some procedures need to be met to ensure the reliability of the results. In this study, a Peer Review procedure was followed in order to ensure that a high level of agreement (kappa) was met between two different judges (Creswell, 2003). The final coding was the product of a joint discussion between the two coders involved in the linking process. The level of agreement obtained through a statistical procedure is high, when compared to other studies where the ICF-CY was linked with specific content of extant measures by two independent coders (Nijhuis et al., 2008). However, a limitation of the present study was lack of a third rater to break ties. Having a third, independent, researcher with extensive clinical practice matching the ICF-CY and the CCPSN, would increase reliability 
and clarity tinal coding, leading to more reliable results. Another challenging aspect in the linking process was the definition and identification of meaningful concepts - considering the developmental nature of the CCPSN, this measurement's items are broader and more general than in health related or health status measures, with which other studies of linkage have been conducted. To overcome this, the two coders in the present study defined specific rules to be used as an addendum to the published linking rules (Cieza et al., 2005), and training was conducted with both researchers to ensure application of linkage rules.

It is relevant to underline some limitations and point directions to future research. In order to have access to the functionality profiles of young children with special needs within a biopsychosocial framework, measures of environmental characteristics of children's everyday contexts are needed, as well as its linkage to the environmental factors domain of the ICF-CY. The CCPSN provides data concerning the children's behaviour, characterizing their body functions, activities and participation; it appears to disregard the environmental characteristics, thus omitting the holistic approach of the ICF-CY framework. Further research is needed to fully link assessment aspects to intervention procedures, in a standard language. Another limitation of this study is that it does not consider the role of the ICF-CY universal qualifier. The use of the universal qualifier would allow us to describe the magnitude of the assessed needs, and decide for intervention goals with basis on such description. In order to achieve this, further studies are necessary to map the universal qualifier scale with the CCPSN and other instruments' rating scales, so as to provide guidelines for effective assessment-intervention procedures. Furthermore, given the ICF-CY's current implementation in several countries, namely in Portugal, where its use is mandated by law in special education and early childhood intervention services, future research would benefit from the following considerations: (a) clinicians with recognized expertise in developmental issues should be involved, in order to test the validity of the mapping process, experienced; (b) developing a data base that illustrates the mapping procedure between extant measures and ICF dimensions, thus facilitating the assessment-intervention process by professionals delivering services; (c) using assessment tools that describe children's functioning based on the ICFCY mapped content, could be helpful in achieving a systemic approach to ECI team work, through the adoption of a transdisciplinary narrative.

Lastly, the Kappa values in the present study were highly variable in the different domains, resulting in low mean Kappa values. Considering the percentage of agreement, although the level of disagreement between the two coders $(28 \%)$ is lower than in other studies (e.g. Nijhuis et al., 2008), it shows that often researchers may not have the same understanding regarding some ICFCY functionality dimensions. This may be remedied by operationally defining some of the ICF-CY dimensions. For instance, while activity and participation dimensions such as learning through pretend play (d1314) and learning through symbolic play (d1314) had a Kappa level of 1 , (highest possible agreement), other dimensions such as acquiring skills (d155) or solving problems (d175) obtained a Kappa value of 0 , meaning there was no agreement in the matching of these specific codes. It is of our opinion that some ICF- 
CY dimensions are very well defined and clearly framed within theoretical grounds (e.g., learning through symbolic play), while other dimensions need further theoretical framing regarding their scope, or specification of their definitions (e.g., acquiring skills). According to the Director of the Division of Mental Health, Norman Sartorius, (World Health Organization, 1993) "A classification is a way of seeing the world at a point in time." When applied to the ICF-CY, we can interpret this tool as a changing procedure, needing further reviews to improve its utility for education and health purposes. This need for a clearer definition of the ICF-CY dimensions may also have contributed to the level of disagreement between the coders. Further research involving professionals from diverse disciplines (e.g., teachers, physicians, psychologists, therapists, etc.) working in different settings (e.g., schools, clinics) would be helpful in clarifying the ICF-CY definitions, from a theoretical and practice standpoint. Such clarification may lead to higher levels of inter-rater agreement when mapping content onto the ICF-CY classification taxonomy.

\section{Summary}

The purpose of this study was to explore resources to improve the assessment-intervention-process for young children with special needs. The Biopsychosocial Model of development, along with the International Classification of Functionality, Disability and Health for Children and Youth (ICF-CY; WHO, 2007), provide a theoretical framework, as well as a classification system, that enable the documentation of functionality profiles of children using a common language across settings and disciplines. The Carolina Curriculum for Preschoolers with Special Needs (CCPSN; Johnson-Martin et al., 1990) is a curriculum based assessment measure, which provides information both for the assessment of children's behaviour as well as for planning interventions. In line with the World Health Organization's recommendations for developing correspondences between assessment measures and the ICF-CY classification system, this study presents results of the linkage between ICF-CY and CCPSN, using deductive content analysis and the previously published linking rules (Cieza et al., 2005). Results show a moderate level of reliability based on inter-rater agreement between the coders who developed the linkage process, as measured by Kappa Cohen's coefficient. In addition, we found that the CCPSN covers body functions as well as activities and participation considered in the ICF$\mathrm{CY}$, but does not cover the environmental factors or body structures domains.

\section{Acknowledgements}

The authors wish to thank all the professionals involved in the study for providing information regarding measures of assessment in preschool settings.

\section{References}

Bagnato, SJ, Neisworth, JT, Pretti-Frontczak, K (2010). LINKing authentic assessment and early childhood intervention: Best measures for best practices (4th Edition). Baltimore, MD: Paul Brookes Publishing Co., Inc.

Bronfenbrenner, U. (2001). The bioecological theory of human development. In: N.J. 
Smelser and P. B. Baltes (Eds.). International encyclopedia of the social and behavioral sciences, Vol. 10, pp. 6963- 69709. New York: Elsevier.

Bronfenbrenner, U. and Morris, P.A. (1998). The ecology of developmental processes. In: W. Damon and R.M. Lerner (Eds) "Handbook of child psychology, Vol. 1: Theoretical models of human development (5th Edition). pp993-1023. New York: John Wiley and Sons, Inc.

Cieza, A., Geyh, S., Chatterji, S., Kostanjsek, N., and Üstün, B. (2005). ICF linking rules: an update based on lessons learned. Journal of Rehabilitation Medicine, 37(4), 212-218. Retrieved from E-Journals database.

Cohen, J. (1960). A Coefficient agreement for nominal scales. Educational and Psychological Measures, 20, 37-46.

Creswell, J.W. (2003). Research Design: Qualitative, Quantitative and Mixed Methods approaches. SAGE publications.

Elo, S. and Kyngas, H. (2007). The qualitative content analysis process. Journal of advanced nursing, 62 (1), 107-115.

Frankel, R.M., Quill, T.E. and McDaniel, S.H. (2003). The biopsychosocial approach: Past, present, future. University of Rochester Press. Rochester, NY.

Johnson-Martin, N.M., Attermeier, S. and Hacker, B. (1990). North Carolina Curriculum for preschoolers 2 to 5 years old.

Landis, J.R. and Koch, G.G. (1977). The measurement of observer agreement for categorical data. Biometrics, 33, 159-174.

Lollar, D. and Simeonsson. R. (2005). Diagnosis to Function: Classifications for children and Youths. Developmental and Behavioral Pediatrics, 26, 323-330.

McCartney, K. and Rosenthal, R. (2000). Effect size, practical importance, and social policy for children. Child Development, 71, 173-180.

Nijhuis, B.J.G., Reinders-Messelink, H.A., de Blecourt, A.C.E., Boonstra, A.M., Calame, E.H.M., Groothoff, J.W., Nakken, H. and Postema, K. (2008). Goal setting in Dutch paediatric rehabilitation. Are the needs and principal problems of children with cerebral palsy integrated into their rehabilitation goals? Clinical Rehabilitation, 22, 348-363.

Sameroff, A.J. (2009). The Transactional Model of Development: How Children and Contexts Shape Each Other. Washington, DC: American Psychological Association.

Sameroff, A. J. and Chandler, M.J. (1975). Reproductive risk and the continuum of caretaking casualty. In F. D. Horowitz, M. Hetherington, S. Scarr- Salapatek and G. Siegel (Eds.), * Review of child development research" Vol. 4, pp. 83-104. Hillsdale, NJ: Lawrence Erlbaum Associates.

Simeonsson, R.J. (2006). Appendix C: Defining and Classifying Disability in Children. In Field M.J., Jette A., and Martin, L. Workshop on Disability in America: A New Look. Washington, DC: National Academy Press. pgs.67-87.

Starrost, K., Geyh, S., Trautwein, A., Grunow, J., Ceballos-Baumann, A., Prosiegel, M. and Cieza, A. (2008). Interrater reliability of the Extended ICF Core Set for Stroke applied by physical therapists. Physical Therapy, 88 , 841-856.

World Health Organization (1994). International Statistical Classification of Diseases and Related Health Problems. 10th ed. Geneva, Switzerland.

World Health Organization (2001). International Classification of Functioning, Disability, and Health: ICF. Genéve, Switzerland.

World Health Organization (2007). International Classification of Functioning, Disability, and Health-Version for Children and Youth: ICFCY. WHO Workgroup for Development of Version of ICF for Children and Youth.

Xiong, T. and Hartley, S. (2008). Challenges in linking health-status outcome measures and clinical assessment tools to the ICF. Advances in Physiotherapy, 10, 152-156. 